\title{
Organ-Sparing Strategies in Muscle-Invasive Bladder Cancer
}

\section{J Alfred Witjes \\ Astrid AH Feikema}

Department of Urology, Radboudumc

Nijmegen, The Netherlands
Correspondence: J Alfred Witjes Department of Urology (6I0),

P.O. Box 910I, 6500 HB Nijmegen, The Netherlands

Tel +3I 24 36I67/2

Fax +3। 24 354I03।

Email Fred.witjes@radboudumc.nl
Abstract: Radical cystectomy (RC) is the treatment of choice and is strongly recommended for patients with pT2-4aN0M0 bladder cancer in both the European Association of Urology (EAU) and American Urological Association (AUA) muscle-invasive bladder cancer (MIBC) guidelines. RC is a challenging operation, with significant perioperative and postoperative morbidity and mortality, having short-term complication rates between $14.4 \%$ and $21.7 \%$, and long-term oncological survival rates ranging from $60 \%$ after 5 years to $43 \%$ after 10 years. The impact on quality of life $(\mathrm{QoL})$ in patients after treatment for bladder cancer is significantly worse than in other pelvic cancers. Although RC is strongly recommended as the gold standard, there is a need for bladder-sparing options in MIBC. Attempts to improve mortality and morbidity rates after RC have been made by implementing Enhanced Recovery After Surgery (ERAS), robot-assisted RC, and sexual function-preserving techniques. None of these significantly improves QoL or functional outcome. Because of the invasiveness of $\mathrm{RC}$, other therapeutic options have been evaluated. Transurethral resection of the bladder tumor (TURB) plays an important role in the diagnostic evaluation of MIBC and has also been reviewed as a curative option, although the oncological results appear inferior to RC. Furthermore, improved radiotherapy (RT) and widely used chemotherapy, both as monotherapeutic options in bladder cancer, are not as effective as radical surgery, with lower survival rates. Trimodality treatment (TMT) in bladder cancer combines TURB with chemotherapy and RT. The goal of TMT is preserving the bladder and QoL without compromising oncological outcome. A 2018 review showed no difference in overall survival rates between RC and TMT (30.9\% vs 35.1\%), with lower survival rates after RC than TMT in the first year of follow-up, probably due to higher postoperative mortality. For a selected group of patients, TMT is to be recommended, and it is the most favorable option out of the organsparing strategies in MIBC.

Keywords: bladder cancer, cystectomy, trimodality treatment, oncological outcome, functional outcome, organ preservation

\section{Introduction}

Radical cystectomy (RC) is the standard treatment for certain stages of bladder cancer. For the high-risk group of patients with non-muscle invasive bladder cancer (NMIBC), both the European Association of Urology (EAU) and American Urological Association (AUA) guidelines recommend treatment with bacille Calmette-Guérin (BCG). ${ }^{1,2}$ However, even in these patients RC is carried out. Yafi et al, for example, reported on 2287 RCs performed in Canada between 1998 and 2008, and the distribution of clinical staging was NMIBC in $26.3 \%$ (cTis in $4.8 \%$, cTa in $2.9 \%$, and cT1 in $18.6 \%$ ). ${ }^{3}$ The reasons for performing an $\mathrm{RC}$ include unresectable non-invasive tumors and $\mathrm{BCG}$ 
unresponsiveness. Indeed, the latter situation is one of the reasons for recommending $\mathrm{RC}$. The AUA guideline recommends RC in fit patients with high-grade T1 tumors after BCG induction, or T1 tumors with carcinoma in situ (CIS), lymphovascular invasion (LVI), or variant histologies $(\mathrm{VH}){ }^{2}$ This translates into a moderate recommendation, with grade $\mathrm{C}$ evidence strength. The EAU guideline is more stringent in its recommendation: an immediate $\mathrm{RC}$ should be discussed or even offered to patients in the case of the highest risk of tumor progression (eg, LVI, CIS in the prostatic urethra, or VH) and BCG failures (both recommendations are strong). ${ }^{1}$ In the current era, BCG unavailability may also be a reason to consider $\mathrm{RC}$ in an earlier stage of the disease. The EAU guideline mentions some bladder-sparing alternatives, albeit in patients who are not fit for or who are unwilling to undergo RC: other forms/combinations of intravesical chemotherapy, microwave-induced chemohyperthermia, electromotive drug administration (EMDA), or intravesical or systemic immunotherapy within clinical trials. All of these alternative bladder-sparing strategies are, however, considered oncologically inferior to $\mathrm{RC}$.

At the other end of the scale, in patients with metastatic bladder cancer, $\mathrm{RC}$ is indicated for palliative reasons, such as hematuria of renal obstruction. In many palliative indications, there are bladder-sparing alternatives such as painkillers in case of pain, radiotherapy (RT) in case of hematuria, and drains and catheters in case of obstruction.

In muscle-invasive bladder cancer (MIBC), $\mathrm{RC}$ is strongly recommended for patients with pT2-4aN0M0 bladder cancer in both the EAU and the AUA MIBC guidelines. ${ }^{4,5} \mathrm{RC}$ is thought to have the highest cure rate, although no randomized controlled trial (RCT) has ever been performed comparing $\mathrm{RC}$ with any other form of treatment for MIBC. RC, however, is a major operation, the most challenging operation in oncological urology, with significant perioperative and postoperative morbidity and mortality. Knorr et al published a series of 969 RC operations in 8 years (between 2011 and 2018) in a tertiary care center. ${ }^{6}$ The 30 and 90-day major complication rates were $14.4 \%(n=140)$ and $21.7 \% \quad(n=210)$, respectively, and comorbidity was a predictive factor for complications. Mortality was 9 and 26 in 30 and 90 days, respectively $(0.9 \%$ and $2.6 \%)$. The authors mention that their rates are comparable to the literature, but this was a high-volume center, and minor complications and complications after 90 days were not included. It seems fair to conclude $\mathrm{RC}$ is indeed a major operation, usually carried out in an older patient population with a smoking history and cardiac complaints due to smoking, which is one of the risk factors for bladder cancer.

In the context of long-term complications, 90 days after RC, Hautmann et al presented the grades and rates of long-term complications in 923 neobladders during 25 years in a tertiary center. ${ }^{7}$ Of the 923 patients, 377 (41.8\%) showed long-term complications during a medium followup of 72 months. Most of the reported complications were diversion related, varying from tumor- and non-tumorrelated ureteroileal stenosis $(11.05 \%)$ to incisional hernia after a midline transperitoneal incision (4.4\%), febrile urinary tract infection (UTI) due to failed neobladder emptying (13.6\%), and metabolic complications with sodium substitution in $33 \%$ of the patients 1 year after surgery. Furthermore, rare and unexpected complications occurred, including neovesicointestinal fistula, neobladder rupture, reservoir stone formation, and subneovesical obstruction. In conclusion, even in the most experienced hands the long-term complication rates of $\mathrm{RC}$ and neobladder formation are not negligible.

In addition to complications of RC in invasive bladder cancer, another non-oncological but important issue after $\mathrm{RC}$ is the impact on quality of life (QoL) after treatment. QoL in patients after bladder cancer is significantly worse than in other pelvic cancers. Sexual problems are commonly reported, together with financial difficulties. ${ }^{8}$ The impact on QoL can be reduced with orthotopic lower urinary tract reconstruction after $\mathrm{RC} .{ }^{9}$ A high percentage of patients $(87 \%)$ with an orthotopic bladder demonstrate unaltered spontaneous and residual-free voiding as well as pad-free continence during both day and night. ${ }^{10}$ However, the use of clean intermittent catheterization (CIC) was still seen in cases with occasional mucus obstruction, residual urine after spontaneous voiding, and hypercontinence. These highly positive functional results came from the category of men with $>20$ years' follow-up, which included the youngest and fittest patients at the time of $\mathrm{RC}$ and the time of the survey presented by Hautmann et al. Therefore, they must be interpreted in the context of some limitations, since these functional results would presumably be worse for low-volume centers or an older patient population with comorbidities.

When critically evaluating complications and impact on QoL caused by $\mathrm{RC}$, we have to put this operation into context with regard to oncological results and survival in the evaluation of long-term clinical outcomes. For instance, a group of 1054 patients treated with RC for bladder cancer was presented by Stein et al. ${ }^{9}$ The 
recurrence-free survival and overall survival (OS) for all patients at 5 years was $68 \%$ and $66 \%$, and $60 \%$ and $43 \%$ at 10 years, respectively. Invasion of the muscle layer by the cancer is directly correlated with an increased pathological stage and higher chance of lymph-node-positive disease. Both are associated with higher recurrence rates and worse survival. Survival for patients with lymphnode-positive disease was $35 \%$ and $34 \%$ at 5 years, and $31 \%$ and $23 \%$ at 10 years. Therefore, pathological stage is an important determinant of survival in patients undergoing RC.

Although RC is strongly recommended in guidelines as the treatment of choice for MIBC, there are clear issues such as short- and long-term complications, functional outcomes, and impact on QoL, as well as limited survival in spite of this radical treatment, and there has been no improvement in survival of bladder cancer patients for decades. ${ }^{11}$ All in all, there seems to be room for improvement in this guideline treatment of choice.

The objective of this report is to review different organ-sparing strategies and treatment options in patients with non-metastatic MIBC, comparing oncological and functional outcomes from different organ-sparing strategies with the current treatment of choice recommended by different MIBC guidelines.

\section{How to Decrease or Improve Morbidity After RC?}

Several attempts have been made to improve early recovery since the introduction of $\mathrm{RC}$ in bladder cancer patients. One of these is the introduction of Enhanced Recovery After Surgery (ERAS) as a perioperative approach for patients undergoing major abdominal surgery, such as RC. ERAS is a multimodal concept aiming to reduce surgical stress and facilitate postoperative recovery. ${ }^{12}$ These protocols contain multiple key elements such as preoperative counseling, analgesic regimens, early mobilization after surgery, and avoidance of a nasogastric tube. While ERAS protocols attempt to reduce morbidity after $\mathrm{RC}$, their implementation has been limited or unavailable. In 2020, Williams et al presented a systematic review identifying 11 studies with 2077 patients from six centers regarding ERAS protocols and RC outcomes. ${ }^{13}$ ERAS pathways for patients undergoing $\mathrm{RC}$ reduced the length of hospitalization, time to recovery of bowel function, and complications, with no increase in mortality.
In addition to striving for early recovery using the multimodal approach of ERAS, a lot of research has analyzed the differences in surgical approach in $\mathrm{RC}$, ie, open radical cystectomy (ORC) versus robot-assisted radical cystectomy (RARC). For several decades, since the introduction of robotic surgery, the use of RARC has gained popularity as surgical treatment in bladder cancer. This increasing popularity is probably due to its improved ergonomics, potentially decreased morbidity, and the reduction of postoperative complications. Still, the question remains as to whether RARC is inferior to ORC in the treatment of bladder cancer.

Soria et al evaluated the perioperative as well as 30and 90-day complication rates of RARC versus ORC in a large multicenter cohort of 1197 patients treated for bladder cancer with RC at 25 institutions between 1971 and 2015. ${ }^{14}$ The choice of surgical technique was based on surgeon's preference, institutional policy, and patient and tumor characteristics. RARC was associated with reduced blood loss, increased operation time, and shorter length of stay. Conversely, RARC was also associated with an increased likelihood of readmission. However, there were no differences in complication rate, reoperations, or mortality between these surgical approaches. This evidence is supported by Sathianathen et al, who reviewed five RCTs comparing RARC to ORC. ${ }^{15}$ They supported the findings of a reduction in blood loss and a reduction in length of stay, both of which were clinically insignificant. Their results support the idea that the individual surgeon is more important than his or her tools. Therefore, it is safe to say that there are no apparent differences in safety and morbidity between ORC and RARC, despite the growing popularity of RARC. Both surgical techniques are largely comparable in terms of oncological outcomes, complication rates, and QoL outcomes.

Wijburg et al, in 2021, published their multicenter comparative effectiveness study comparing RARC versus ORC in terms of 90-day complications, health-related QoL, and clinical outcomes. ${ }^{16}$ The study showed no statistically significant differences between RARC and ORC in terms of effectiveness, therefore supporting the results of previous RCTs and systematic reviews.

In light of improving functional outcome in patients undergoing $\mathrm{RC}$, there are different sexual functionpreserving surgical techniques which focus on improving sexual and voiding function. Technical variations range from prostate-sparing $\mathrm{RC}$ to other procedures where only capsule, seminal vesicle, or neurovascular bundles are spared 
during cystectomy. The evidence for these surgical techniques suggests that they may yield better sexual outcomes in male patients than standard $\mathrm{RC}$, without compromising oncological outcomes. ${ }^{17}$ However, the quality of evidence is low for sexual-preserving cystectomy techniques in men. The same was investigated in a study by Veskimäe et al in women, comparing pelvic organ-preserving radical cystectomy (POPRC), such as neurovascular bundle-preserving, vagina-preserving, or genitalia-sparing variations, with standard RC. ${ }^{18}$ For a select group of patients, POPRC may potentially be comparable to standard $\mathrm{RC}$ for oncological outcomes while improving the quality of sexual life. However, well-designed RCTs and prospective multicenter studies with valid data collection are needed before these organ-sparing surgical techniques can be implemented in the treatment of MIBC.

\section{Bladder Sparing}

As stated in the Introduction, $\mathrm{RC}$ is strongly recommended in the treatment of MIBC. But since the invasiveness of this surgical treatment comes with challenges in terms of complications and functional outcomes, other therapeutic options are evaluated.

Transurethral resection of the bladder (TURB) plays an important role in the diagnostic evaluation of MIBC since it enables histopathological diagnosis and staging. ${ }^{4}$ In 2001, Herr reported the 10-year outcome of radical TURB as monotherapy in the case of MIBC in 151 patients with a reTURB without invasive tumor (T0 or T1). ${ }^{19}$ Of these patients, 99 received TURB as definitive therapy and 52 had $\mathrm{RC}$, with a 10 -year disease-specific survival (DSS) of $76 \%$ and $71 \%$, respectively. Of note, patients with $\mathrm{T} 1$ on reTURB had worse outcome than those with T0 ( $82 \%$ vs $57 \%$ survival, $p=0.003$ ). Moreover, 34 of these 99 patients had a new invasive bladder tumor, of which 18 were salvaged with cystectomy, whereas 16 died of the disease. Herr concluded that radical TURB for MIBC was a successful bladder-sparing strategy in selected patients who have no residual tumor on repeat vigorous resection of the primary site. In line with Herr, a prospective study by Solsona et al analyzed the oncological outcomes of 133 patients with MIBC undergoing radical TURB as monotherapy with negative biopsies after restaging. ${ }^{20}$ The bladder was preserved, with progression-free survival of $75.5 \%, 64.9 \%$, and $57.8 \%$ at 5,10 , and 15 years, respectively. In the 15 -year follow-up period, approximately $30 \%$ of the patients experienced disease progression, of which $7.7 \%$ was associated with metastasis. However, progression was associated with a high mortality rate of $67.5 \%$. The authors concluded that TURB as monotherapy in MIBC can be a reliable therapeutic approach after complete tumor resection, including negative biopsies in the tumor bed, and this approach can be considered an option when patients are unfit for or do not favor cystectomy. Still, TURB alone as a curative option is not recommended since it requires meticulous patient selection and information, as well as persistent bladder follow-up, and the oncological results appear to be inferior to $\mathrm{RC}^{4}$

With improvements in external beam radiotherapy (EBRT) techniques, there has been renewed interest in EBRT for the treatment of bladder cancer. These techniques result in better coverage of the target organ (bladder and possibly nodes) with lower doses to the surroundings. Hafeez et al, for example, reported on 55 patients who were unfit for cystectomy, of whom 48 completed EBRT with acceptable toxicity, with only $17 \%$ local progression after 2 years. $^{21}$ These results show good local control with acceptable toxicity. Prognostic factors for EBRT are well known: tumor size, presence of hydronephrosis or CIS, and radicality of the initial TURB. In spite of this, however, EBRT monotherapy has been surpassed by the improved outcomes after trimodality bladder-preserving treatment (or trimodality therapy, TMT), as will be discussed in the next section. In all, in spite of technical improvements in EBRT, EBRT monotherapy is not as effective as radical surgery or TMT.

Chemotherapy is widely used as neoadjuvant therapy in bladder cancer. Although chemotherapy in the neoadjuvant setting is able to result in a pT0 status after cystectomy (an event sometimes even used as a surrogate endpoint in trials), chemotherapy as monotherapy seldom results in long-term remission. A large retrospective analysis of a National Cancer Database cohort showed a 5 -year OS of $32.9 \%$ (36.2\% in stage cT2) in over 1500 patients treated with multiagent chemotherapy after TURB. ${ }^{22}$ Since this is clearly less than with radical surgery or TMT, chemotherapy monotherapy is also not recommended as standard treatment in bladder cancer.

Another bladder-sparing technique considered is partial cystectomy (PC). Despite renewed interest in bladdersparing techniques, the EAU guideline does not mention PC. The AUA considers PC as a therapeutic option in a highly select group of patients, where the selection criteria include accessible tumor location, size $<3 \mathrm{~cm}$, no multifocal CIS, no hydronephrosis, adequate bladder function, and no residual T1 or higher stage disease. ${ }^{5}$ The most recent review, by Knoedler et al, summarizes the literature regarding $\mathrm{PC}$ for bladder cancer in terms of utilization, 
outcomes, recurrence, and complications of PC. ${ }^{23}$ About $7-10 \%$ of all cystectomies in the USA are performed partially. With regard to oncological outcomes, no difference was found between RC and PC in 10-year metastasisfree survival $(61 \%$ vs $66 \%)$ or cancer-specific survival ( $58 \%$ vs $63 \%$ ). These numbers only apply to appropriately selected patients. Close surveillance after PC is necessary since the median time from PC to salvage RC was 1.6 years owing to early recurrence. Of these patients, $61.2 \%$ had organ-confined disease, whereas $19.4 \%$ had extravesical disease and $19.4 \%$ had lymph-node metastasis. Current data indicate that $15.8 \%$ of patients experience an inhospital complication, with a mortality rate of $1.8 \%$. Those patients who died had higher comorbidity indices. It is likely that younger, healthier patients are more likely to undergo RC. For medically unfit patients, $\mathrm{PC}$ can be offered as a therapeutic option, but there are no RCTs consisting of head-to-head trials on PC as a beneficial treatment in MIBC.

\section{Trimodality Bladder-Preserving Treatment}

Trimodality bladder-preserving treatment (TMT) in bladder cancer combines TURB with chemotherapy and RT. The goal of TMT is preserving the bladder and QoL without compromising oncological outcome. The rationale behind combining TURB with RT is to achieve maximal local tumor control in the bladder and adjacent nodes. The addition of systemic chemotherapy has the potential to improve locoregional control since it works as a radiosensitizer, and not specifically to address micrometastases, which are targeted by (neo)adjuvant platinumbased combination chemotherapy. ${ }^{4,5}$

Maximal resection of the visible tumor with TURB is the first step in TMT. In this case, patient selection is critical, meaning patients with $\mathrm{T} 2$ tumors, good bladder function, and no CIS. Even in the case of an initial presumed complete resection, a second TURB has been suggested to reveal tumor in more than $50 \%$ of patients. In addition to TURB, a conventional radiation schedule includes radiation fields of the pelvis (with bladder and/or bladder tumor boost), bladder only, or partial bladder only, with an initial dose of 40-45 Gy. Concurrently with RT, different chemotherapy regimens are used in TMT, but most evidence exists for cisplatin and mitomycin C plus 5-fluorouracil (5-FU). Adding chemotherapy with RT may sensitize tumor cells to radiation, thus increasing cell kill in a synergistic fashion. This has the potential to improve locoregional control, and may provide additional benefit for the control of occult metastatic disease at an early stage.

The question of whether TMT has the same oncological outcomes as RC remains unanswered, since no randomized trials have been published on the treatment of MIBC. Five-year OS rates vary from $36 \%$ to $74 \%$, respectively, with salvage cystectomy rates of $10-30 \%$. A small retrospective study by Kulkarni et al showed that TMT in 56 patients provided midterm survival outcomes comparable to RC in selected bladder cancer cases. ${ }^{24}$ Patients suitable for TMT had solitary tumors $<5 \mathrm{~cm}$, minimal to no hydronephroses, good bladder function, and no CIS. The median follow-up was 4.51 years, with 20 deaths in the $\mathrm{RC}$ group and 22 deaths in the TMT group ( $p=0.84)$. The 5-year DSS rates for RC and TMT were $73.2 \%$ and $76.6 \%$, respectively $(p=0.49)$. Salvage cystectomy was performed in six $(10.7 \%)$ of the 56 patients treated with TMT. This study demonstrates the excellent and comparable results of TMT in selected patients with MIBC, but has some limitations, including a selection bias based on clinical factors and the small number of patients (112), with a relatively short follow-up period of 4.51 years.

To address the overarching question of OS in TMT versus RC, Kaushik et al conducted a large propensity score-weighted comparative analysis using the National Cancer Database. ${ }^{25}$ Data from 15,854 patients who received RC and 4050 patients who were treated with TMT were available for analysis. The median OS for the entire cohort was 30.5 months, and was longer in those who were treated with $\mathrm{RC}$ than in the patients receiving TMT (36.2 vs 24.2 months). The 5-year OS for RC was $40.4 \%$ versus $29.4 \%$ in patients receiving TMT. Covariates associated with a decreased OS included advanced age, higher comorbidity scores, T3 and T4 stages versus T2, and positive lymph nodes. An interesting and significant interaction of treatment type and time was identified: OS was shorter after RC than TMT in the first year follow-up. This increased risk may partly be related to higher complication and mortality rates following RC.

Another, more recent, large systematic review and metaanalysis on oncological outcomes after TMT versus RC was conducted by Fahmy et al. ${ }^{26}$ They extracted data on 3402 patients treated with TMT and 26,891 patients with RC, all diagnosed with MIBC. For the entire cohort, the OS and DSS were not significantly different between RC and TMT: 10-year OS $30.9 \%$ vs $35.1 \%$ and 10 -year DSS $50.9 \%$ vs $57.8 \%$, respectively. Approximately $75 \%$ achieved complete response 
to TMT. Complete responders had a lower risk of undergoing $\mathrm{RC}$ during follow-up. With regard to recurrence after treatment, $\mathrm{RC}$ resulted in lower recurrence rates, which may suggest that the bladder remains a potential source of disease recurrence. Comparing oncological outcomes from TMT with RC remains difficult, considering, among other factors, differences in patient selection. There are no successfully completed RCTs comparing the outcomes of TMT with RC. Reviewing the available studies regarding bladder-preserving strategies such as TURB, EBRT, chemotherapy, and PC as monotherapy provides no strong evidence for their use as an alternative to RC in MIBC. In addition, no high-quality evidence is available comparing QoL between the different treatment options. TMT seems to be an option in patients with MIBC who are unfit for RC. Recently available data, ${ }^{24-26}$ however, suggest that the long-term oncological outcome after TMT may be comparable to that after RC, and TMT may, therefore, be a valid alternative in the treatment of MIBC. One of the main arguments for TMT in operable patients is to avoid functional drawbacks regarding sexual and voiding function. However, there are not enough data regarding TMT and QoL after bladder-preserving treatment compared to $\mathrm{RC}$.

In conclusion, a trimodal bladder-preserving treatment is to be recommended in patients with MIBC who are medically unfit for $\mathrm{RC}$, or those who desire bladder preservation because of possible functional drawbacks after RC.

\section{Disclosure}

The authors report no conflicts of interest in this work.

\section{References}

1. Babjuk M, Burger M, Compérat EM, et al. European Association of Urology Guidelines on Non-muscle-invasive Bladder Cancer (TaT1 and Carcinoma In Situ) - 2019 Update. Eur Urol. 2019;76(5):639-657. doi:10.1016/j.eururo.2019.08.016

2. Chang SS, Boorjian SA, Chou R, et al. Diagnosis and Treatment of Non-Muscle Invasive Bladder Cancer: AUA/SUO Guideline. $J$ Urol. 2016;196(4):1021-1029. doi:10.1016/j.juro.2016.06.049

3. Yafi FA, Aprikian AG, Chin JL, et al. Contemporary outcomes of 2287 patients with bladder cancer who were treated with radical cystectomy: a Canadian multicentre experience. BJU Int. 2010;108(4):539-545. doi:10.1111/j.1464-410X.2010.09912.x

4. Witjes JA, Bruins HM, Cathomas R, et al. European Association of Urology Guidelines on Muscle-invasive and Metastatic Bladder Cancer: summary of the 2020 Guidelines. Eur Urol. 2021;79 (1):82-104. doi:10.1016/j.eururo.2020.03.055

5. Chang SS, Bochner BH, Chou R, et al. Treatment of Non-Metastatic Muscle-Invasive Bladder Cancer: AUA/ASCO/ASTRO/SUO Guideline. J Urol. 2017;198(3):552-559. doi:10.1016/j.juro.2017. 04.086
6. Knorr JM, Ericson KJ, Zhang JJH, et al. Comparison of major complications at 30 and 90 days following radical cystectomy. Urology. 2021;148:192-197. doi:10.1016/j.urology.2020.08.038

7. Hautmann RE, de Petriconi RC, Volkmer BG. 25 Years of Experience With 1000 Neobladders: long-Term Complications. J Urol. 2011;185 (6):2207-2212. doi:10.1016/j.juro.2011.02.006

8. Catto JWF, Downing A, Mason S, et al. Quality of life after bladder cancer: a cross-sectional survey of patient-reported outcomes. Eur Urol. 2021;79(5):621-632. doi:10.1016/j.eururo.2021.01.032

9. Stein JP, Lieskovsky G, Cote R, et al. Radical cystectomy in the treatment of invasive bladder cancer: long-term results in 1054 patients. J Clin Oncol. 2001;19(3):666-675. doi:10.1200/JCO.2001.19.3.666

10. Hautmann RE, Volkmer B, Egghart G, et al. Functional Outcome and Complications following Ileal Neobladder Reconstruction in Male Patients without Tumor Recurrence. More than 35 Years of Experience from a Single Center. J Urol. 2021;205(1):174-182. doi:10.1097/JU.0000000000001345

11. Quaresma M, Coleman MP, Rachet B. 40-year trends in an index of survival for all cancers combined and survival adjusted for age and sex for each cancer in England and Wales, 1971-2011: a populationbased study. Lancet. 2015;385(9974):1206-1218. doi:10.1016/ S0140-6736(14)61396-9

12. Cerantola Y, Valerio M, Persson B, et al. Guidelines for perioperative care after radical cystectomy for bladder cancer: Enhanced Recovery After Surgery $\left(\right.$ ERAS $\left.^{\circledR}\right)$ society recommendations. Clin Nutrition. 2013;32(6):879-887. doi:10.1016/j.clnu.2013.09.014

13. Williams SB, Cumberbatch MGK, Kamat AM, et al. Reporting Radical Cystectomy Outcomes Following Implementation of Enhanced Recovery After Surgery Protocols: a Systematic Review and Individual Patient Data Meta-analysis. Eur Urol. 2020;78 (5):719-730. doi:10.1016/j.eururo.2020.06.039

14. Soria F, Moschini M, D'andrea D, et al. Comparative Effectiveness in Perioperative Outcomes of Robotic versus Open Radical Cystectomy: results from a Multicenter Contemporary Retrospective Cohort Study. Eur Urol Focus. 2020;6(6):1233-1239. doi:10.1016/j.euf.2018. 11.002

15. Sathianathen NJ, Kalapara A, Frydenberg M, et al. Robotic assisted radical cystectomy vs open radical cystectomy: systematic review and meta-analysis. $J$ Urol. 2019;201(4):715-720. doi:10.1016/j. juro.2018.10.006

16. Wijburg CJ, Michels CTJ, Hannink G, et al. Robot-assisted Radical Cystectomy Versus Open Radical Cystectomy in Bladder Cancer Patients: a Multicentre Comparative Effectiveness Study. Eur Urol. 2021;79(5):609-618. doi:10.1016/j.eururo.2020.12.023

17. Hernández V, Espinos EL, Dunn J, et al. Oncological and functional outcomes of sexual function-preserving cystectomy compared with standard radical cystectomy in men: a systematic review. Urol Oncol. 2017;35(9):539.e17-539.e29. doi:10.1016/j.urolonc.2017.04.013

18. Veskimäe E, Neuzillet Y, Rouanne M, et al. Systematic review of the oncological and functional outcomes of pelvic organ-preserving radical cystectomy (RC) compared with standard RC in women who undergo curative surgery and orthotopic neobladder substitution for bladder cancer. BJU Int. 2017;120(1):12-24. doi:10.1111/bju.13819

19. Herr HW. Transurethral Resection of Muscle-Invasive Bladder Cancer: 10-Year Outcome. J Clin Oncol. 2001;19(1):89-93. doi:10.1200/JCO.2001.19.1.89

20. Solsona E, Iborra I, Collado A, Rubio-Briones J, Casanova J, Calatrava A. Feasibility of radical transurethral resection as monotherapy for selected patients with muscle invasive bladder cancer. J Urol. 2010;184(2):475-481. doi:10.1016/j.juro.2010.04.008

21. Hafeez S, McDonald F, Lalondrelle S, et al. Clinical Outcomes of image guided adaptive hypofractionated weekly radiation therapy for bladder cancer in patients unsuitable for radical treatment. Int J Radiation Oncol Biol Phys. 2017;98(1):115-122. doi:10.1016/ j.ijrobp.2017.01.239 
22. Audenet F, Waingankar N, Ferket BS, et al. Effectiveness of transurethral resection plus systemic chemotherapy as definitive treatment for muscle invasive bladder cancer in population level data. J Urol. 2018;200(5):996-1004. doi:10.1016/j.juro.2018.06.001

23. Knoedler JJ, Boorjian SA, Kim SP, et al. Does Partial Cystectomy Compromise Oncologic Outcomes for Patients with Bladder Cancer Compared to Radical Cystectomy? A Matched Case-Control Analysis. J Urol. 2012;188(4):1115-1119. doi:10.1016/j.juro.2012. 06.029

24. Kulkarni GS, Hermanns T, Wei Y, et al. Propensity score analysis of radical cystectomy versus bladder-sparing trimodal therapy in the setting of a multidisciplinary bladder cancer clinic. J Clin Oncol. 2017;35(20):2299-2305. doi:10.1200/JCO.2016.69.2327
25. Kaushik D, Wang H, Michalek J, et al. Chemoradiation Vs Radical Cystectomy for Muscle-invasive Bladder Cancer: a Propensity Score-weighted Comparative Analysis Using the National Cancer Database. Urology. 2019;133:164-174. doi:10.1016/j.urology.2019.05.062

26. Fahmy O, Khairul-Asri MG, Schubert T, et al. A systematic review and meta-analysis on the oncological long-term outcomes after trimodality therapy and radical cystectomy with or without neoadjuvant chemotherapy for muscle-invasive bladder cancer. Urol Oncol. 2018;36(2):43-53. doi:10.1016/j.urolonc.2017.10.002

\section{Publish your work in this journal}

Cancer Management and Research is an international, peer-reviewed open access journal focusing on cancer research and the optimal use of preventative and integrated treatment interventions to achieve improved outcomes, enhanced survival and quality of life for the cancer patient.
The manuscript management system is completely online and includes a very quick and fair peer-review system, which is all easy to use. Visit http://www.dovepress.com/testimonials.php to read real quotes from published authors. 